\title{
Electrochromic Solid-State Devices Using Organic-Metallic Hybrid Polymers
}

\author{
Masayoshi Higuchi · Yumeno Akasaka · \\ Taichi Ikeda $\cdot$ Akari Hayashi $\cdot$ Dirk G. Kurth
}

Received: 8 October 2008/ Accepted: 3 December 2008/Published online: 23 December 2008

(C) Springer Science+Business Media, LLC 2008

\begin{abstract}
Organic-metallic hybrid polymers are formed by the complexation of metal ions with organic modules bearing two coordination sites. The hybrid polymers consisting of bis-terpyridines and metal ions such as Fe(II) or $\mathrm{Ru}$ (II) have a specific color based on the metal-to-ligand charge transfer (MLCT) absorption. Cyclic voltammograms of the polymers exhibit a reversible redox wave according to the redox reaction of the metal ions. Interestingly, a polymer film cast on an indium tin oxide (ITO) electrode exhibits excellent electrochromic properties; the color of the film disappears when a higher potential as compares to the redox potential of the metal ions is applied to the polymer film. In addition, multicolor electrochromic changes appear on introducing two types of metal ions to the polymer. Electrochromic solid-state devices are successfully fabricated by using these polymers.
\end{abstract}

This paper is dedicated to Professor Takakazu Yamamoto in honor of his pioneering research efforts and accomplishments in the fields of organometallics and $\pi$-conjugated polymers.

M. Higuchi $(\bowtie) \cdot$ Y. Akasaka · T. Ikeda · A. Hayashi ·

D. G. Kurth

National Institute for Materials Science (NIMS),

Tsukuba 305-0044, Japan

e-mail: HIGUCHI.Masayoshi@nims.go.jp

\section{Higuchi}

Japan Science and Technology Agency (JST),

Tokyo 102-8666, Japan

D. G. Kurth

Max Plank Institute of Colloids and Interfaces,

Research Campus Golm, 14424 Potsdam, Germany
Keywords Organic-metallic hybrid polymers . Self-assembly · Electrochromic properties . Electronic solid-state devices

\section{Introduction}

$\pi$-Conjugated polymers have received attention for their electrochemical and photochemical properties and applications [1-5], since polyacetylene, one of the $\pi$-conjugated polymers, was discovered to exhibit high electro-conductivity by chemical doping [6-10]. In addition, synthetic methods for synthesizing various $\pi$-conjugated polymers such as poly(bipyridine) were found via coupling reactions in the presence of transition metals [11-15]. $\pi$-conjugated polymers such as polyaniline, polythiophene, polyphenylene, and polypyrrole exhibit electrochromic characteristics (electrochemical changes in color). The electrochromic changes are usually caused by the structural changes in the conjugated polymer according to the electrochemical reduction and oxidation (redox) reactions. The conjugated chain length depends on the polymer structure, which can be modified by using various synthetic methods so that an organic, electrochromic polymer can have different colors. Although such organic polymers with electrochromic properties had been widely examined for their applications about 20 years ago, they were not put to practical use. The main reason for this was the low stability of the materials. As mentioned above, electrochromic characteristics depend on the structural changes in a polymer by the electric injection of charges or electrons. Even if the organic polymer is stable in the presence of light, heat, and air, the oxidative or reductive form with structural changes is often unstable, thereby leading to the deterioration of the material. 
On the other hand, organic-metallic hybrid polymers, which are formed by the complexation of metal ions with organic molecules/polymers bearing coordination sites, are expected to have unique electrochemical, photochemical, magnetic, or catalytic properties based on electronic interactions between organic and metal moieties in the hybrid polymers [16-20]. Furthermore, the properties of the polymers can be enhanced and controlled through a judicious selection of their components. There exist two methods to synthesize hybrid polymers: polymer formation by the complexation of metal ions with organic molecules bearing several coordination sites, or metal ion assembly to organic macromolecules. In the latter case, $\pi$-conjugated polymers with coordination sites, such as polyanilines, polypyridines, and polyazomethines, are often used as the organic macromolecules in the hybrid polymers, because they can trap metal ions in solution and therefore can be regarded as a polymeric ligand [21-24].

Here, we report organic-metallic hybrid polymers synthesized using bis(terpyridyl)benzene as organic molecules bearing several coordination sites [25-29]. Polycomplexation of iron(II), cobalt(II), and ruthenium(II) ions with bis(terpyridyl)benzene results in the formation of metallosupramolecular polyelectrolytes (Fe(II)-MEPE, Co(II)MEPE, Ru(II)-MEPE, and Fe(II)-Ru(II)-MEPE), which belong to a type of organic-metallic hybrid polymers. Thin films of the hybrid polymers exhibit excellent electrochromic properties and can be operated within a reasonable potential range $(\leq 3.0 \mathrm{~V})$. In contrast to organic conjugated polymers, which are well known as common electrochromic materials, the modularity of hybrid polymers offers synthetic simplicity and permits environmentally friendly processing due to its water solubility. We also report the applications of these polymers to electrochromic solid-state devices.

\section{Experimental Section}

\subsection{Materials and General Methods}

1,4-Bis $\left(2,2^{\prime}: 6^{\prime}, 2^{\prime \prime}\right.$-terpyridine-4-yl)benzene, iron(II) acetate $\left(\mathrm{Fe}(\mathrm{OAc})_{2}\right)$, cobalt $(\mathrm{II})$ acetate $\left(\mathrm{Co}(\mathrm{OAc})_{2}\right)$, and tetrakis(dimethylsulfoxide)dichlororuthenium(II) $\left(\mathrm{RuCl}_{2}(\mathrm{DMSO})_{4}\right)$ were purchased from Aldrich or Kokusai Kinzoku Yakuhin Co. Ltd and were used as received. $\mathrm{MeOH}, \mathrm{EtOH}, \mathrm{AcOH}$, $\mathrm{N}, \mathrm{N}$-dimethyl acetamide (DMAc), and ethylene glycol, purchased from Wako or Kanto Chemical Co. In., were dehydrated and were used without further purification. De-ionized $\mathrm{H}_{2} \mathrm{O}$ was used when required. Mass spectra (MS) were measured by using an AXIMA-CFR, Shimadzu/ Kratos TOF Mass spectrometer. High-resolution mass spectra (HRMS) were measured by using a Shimadzu
LCMS-IT-TOF spectrometer. UV/vis spectra were obtained by using a Shimadzu UV-2550 UV-visible spectrophotometer.

\subsection{Electrochemical Measurements}

Voltammetric experiments were performed using a BAS 612B electroanalytical system with a three-electrode cell. Cyclic voltammetry (CV) and amperometric experiments were carried out in argon saturated anhydrous acetonitrile solution containing $0.1 \mathrm{M}$ of tetra- $n$-butylammonium perchlorate (TBAP) as supporting electrolyte by using an electrochemical analyzer, ALS/H $\mathrm{CH}$ instruments, and TBAP and acetonitrile were purchased from Kanto Chemicals. A platinum wire was used as counter electrode, and $\mathrm{Ag} / \mathrm{Ag}^{+}$as reference electrode. The scan rate for $\mathrm{CV}$ analysis was $100 \mathrm{mV} \mathrm{s}^{-1}$. Coated glassy carbon electrode (GCE) $\left(0.07 \mathrm{~cm}^{2}\right)$ or indium tin oxide (ITO) $(10 \mathrm{~mm} \times$ $20-50 \mathrm{~mm} \times 0.5 \mathrm{~mm}, 20 \Omega$ ) samples were used as the working electrode. All the electrodes were purchased from BAS. By using the reference electrode, the ferrocene/ ferrocenium redox couple occurred at $+188 \mathrm{mV}$.

\subsection{General Procedure for the Preparation of $\mathrm{Fe}(\mathrm{II})-, \mathrm{Ru}(\mathrm{II})-$, and $\mathrm{Fe}(\mathrm{II})-\mathrm{Ru}(\mathrm{II})-\mathrm{MEPEs}$}

An equimolar amount of 1,4-bis $\left(2,2^{\prime}: 6^{\prime}, 2^{\prime \prime}\right.$-terpyridine-4yl)benzene and $\mathrm{Fe}(\mathrm{OAc})_{2}$ (or $\left.\mathrm{Co}(\mathrm{OAc})_{2}\right)$ was refluxed in an argon saturated acetic acid (ca. $10 \mathrm{~mL}$ solvent per $\mathrm{mg}$ of 1,4-bis(2,2':6 $6^{\prime}, 2^{\prime \prime}$-terpyridine-4-yl)benzene) for $24 \mathrm{~h}$. The reaction solution was cooled to room temperature and filtered to remove a small amount of insoluble residues. The filtrate was moved to a Petri dish and the solvent was evaporated slowly to dryness. The brittle film was collected and dried further in vacuo overnight to give the corresponding Fe(II)-MEPE (or Co(II)-MEPE) (>90\%).

An equimolar amount of 1,4-bis $\left(2,2^{\prime}: 6^{\prime}, 2^{\prime \prime}\right.$-terpyridine4-yl)benzene and $\mathrm{RuCl}_{2}(\mathrm{DMSO})_{4}$ was refluxed in an argon saturated absolute ethylene glycol (ca. $10 \mathrm{~mL}$ solvent per $\mathrm{mg}$ of 1,4-bis $\left(2,2^{\prime}: 6^{\prime}, 2^{\prime \prime}\right.$-terpyridine-4-yl)benzene) for $24 \mathrm{~h}$. After the reaction solution was cooled to room temperature, THF was added until the solution was colorless. The precipitated polymers were collected by filtration and washed with THF for three times, and then dried in vacuo overnight to give the corresponding $\mathrm{Ru}(\mathrm{II})$-MEPE (95\% quant.).

An organic-metallic hybrid polymer with both $\mathrm{Fe}(\mathrm{II})$ and $\mathrm{Ru}(\mathrm{II})$ ions, $\mathrm{Fe}(\mathrm{II})-\mathrm{Ru}(\mathrm{II})-\mathrm{MEPE}$, was prepared by mixing of a $10 \mathrm{~mL} \mathrm{MeOH}$ solution of $\mathrm{Fe}(\mathrm{II})$-MEPE $(50 \mathrm{mg})$ and a $10 \mathrm{~mL} \mathrm{MeOH}$ solution of $\mathrm{Ru}(\mathrm{II})$-MEPE $(50 \mathrm{mg})$ for $2 \mathrm{~h}$ at room temperature. 
2.4 Preparation of MEPEs Films and Solid-State Electrochromic Devices

About $20 \mu \mathrm{L}$ of a methanol solution (1.0 mg MEPEs/ $0.5 \mathrm{~mL} \mathrm{MeOH}$ ) was cast or spin-coated (500 rpm for $60 \mathrm{~s}$ ) with an ACT 300D spin-coater from ACTIVE, Japan, on Glassy Carbon (GC) $\left(0.07 \mathrm{~cm}^{2}\right.$, BAS $)$ or ITO electrodes $(10 \mathrm{~mm} \times 20-50 \mathrm{~mm} \times 0.5 \mathrm{~mm}, 20 \Omega$, BAS $)$. The methanol was purchased from WAKO pure chemicals.

For the solid-state electrochromic devices, a mixture of polymethylmethacrylate $(7.0 \mathrm{~g})$, propylene carbonate $(20 \mathrm{~mL})$, and $\mathrm{LiClO}_{4}(3.0 \mathrm{~g})$ was used as the polymer gel electrolyte.

\section{Results and Discussion}

\subsection{Synthesis of Fe(II)-, Co(II)-, Ru(II)-, and $\mathrm{Fe}(\mathrm{II})-\mathrm{Ru}(\mathrm{II})-\mathrm{MEPEs}$}

Metallo-supramolecular polyelectrolytes with iron(II) and cobalt(II) ions, Fe(II)-MEPE and Co(II)-MEPE, are synthesized during the complexation of iron(II) acetate and cobalt(II) acetate with 1,4-bis $\left(2,2^{\prime}: 6^{\prime}, 2^{\prime \prime}\right.$-terpyridine-4-yl) benzene, respectively (Fig. 1). Exactly identical molar weights of 1,4-bis(2,2': $6^{\prime}, 2^{\prime \prime}$-terpyridine-4-yl)benzene and iron(II) acetate (or cobalt (II) acetate) are added into acetic acid and refluxed for $24 \mathrm{~h}$. During the reaction, the color of
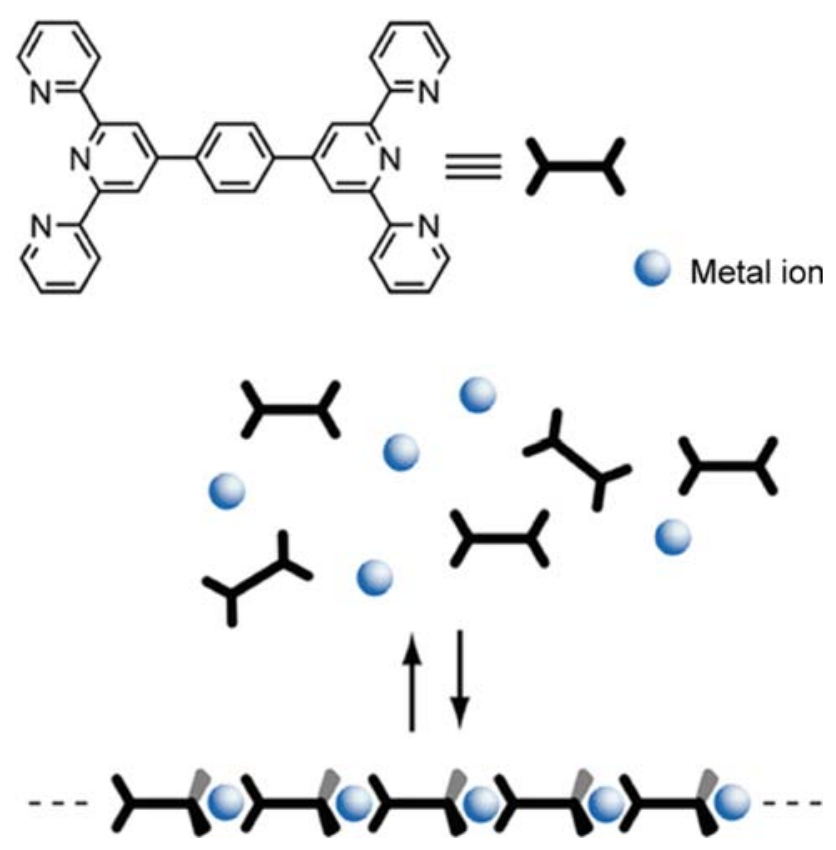

Fig. 1 Metallo-supramolecular polyelectrolytes formed by complexation of 1,4-bis $\left(2,2^{\prime}: 6^{\prime}, 2^{\prime \prime}\right.$-terpyridine-4-yl)benzene and metal ions such as iron(II), cobalt(II), and ruthenium(II) the solution turns purple (or orange) because of the metalto-ligand charge transfer (MLCT) absorption caused by the complexation. After cooling, Fe(II)-MEPE (or Co(II)MEPE) is obtained quantitatively by the evaporation of the solvent. The molar mass of these polymers determined by analytical ultracentrifugation in water was greater than $10^{5} \mathrm{Da}$.

On the other hand, metallo-supramolecular polyelectrolytes with ruthenium(II) ions, Ru(II)-MEPE, did not obtained under the above-mentioned conditions. Finally, we succeeded to synthesize it quantitatively by refluxing in ethylene glycol, which has higher boiling point $\left(197.6^{\circ} \mathrm{C}\right)$ than that $\left(118^{\circ} \mathrm{C}\right)$ of acetic acid.

Polymer blend of Fe(II)-MEPE and Ru(II)-MEPE, $\mathrm{Fe}(\mathrm{II})-\mathrm{Ru}(\mathrm{II})-\mathrm{MEPE}$, is also obtained by mixing of methanol solutions of Fe(II)-MEPE and Ru(II)-MEPE at room temperature.

\subsection{Electrochemistry of Fe(II)-, Co(II)-, Ru(II)-,} and $\mathrm{Fe}(\mathrm{II})-\mathrm{Ru}(\mathrm{II})-\mathrm{MEPEs}$

Fe(II)-MEPE, Co(II)-MEPE, Ru(II)-MEPE are soluble in methanol, and the colors of the solutions are purple, orange, and red, respectively. The electrochemical properties are investigated by means of cyclic voltammetry (CV) using a GC electrode, which is covered with a thin film of Fe(II)-MEPE, as working electrode. Fe(II)-MEPE exhibits a large current response when oxidized in acetonitrile solution. The redox activity is completely reversible $(\Delta E=75 \mathrm{mV}$ at a scan rate of $0.1 \mathrm{~V} / \mathrm{s})$ and $\mathrm{E}_{1 / 2}$ is equal to $0.77 \mathrm{~V}$. Even with Fe(II)-MEPE spin-coated on ITO, a large peak-to-peak separation is not observed; the separation is confined within $100 \mathrm{mV}$. The anodic and cathodic peak currents are proportional to the scan rate, although the redox reaction slows down at a higher scan rate $(0.8 \mathrm{~V} / \mathrm{s})$. Through atomic force microscopy (AFM) measurements, the thickness of the film is estimated to be $250-300 \mathrm{~nm}$. Similarly, thin films of Co(II)-MEPE exhibit a reversible redox peak at $E_{1 / 2}=-0.05 \mathrm{~V}$. The film thickness is estimated to be $300 \mathrm{~nm}$, which is equal to that of $\mathrm{Fe}$ (II)MEPE, as determined from AFM measurements. The redox potential of $\mathrm{Ru}(\mathrm{II})-\mathrm{MEPE}$ is $0.95 \mathrm{~V}$, which is higher than $\mathrm{Fe}$ (II)-MEPE. In cyclic voltammogram of $\mathrm{Fe}(\mathrm{II})-\mathrm{Ru}(\mathrm{II})-$ MEPE, two redox waves at 0.77 and $0.92 \mathrm{~V}$, which are attributed to redox of iron and ruthenium ions in the polymer blend, respectively, are observed.

\subsection{Electrochromic Properties of Fe(II)-MEPE}

We note a strong color change from blue (reduced) and colorless (oxidized) during the cyclic voltammetry of Fe(II)-MEPE spin-coated on ITO electrode. Even after 500 switching cycles we do not detect any sign of fatigue. The 
color change is readily observed by visual inspection even at a scan rate of $800 \mathrm{mV} / \mathrm{s}$ indicating a fast response. The oxidized $\mathrm{Fe}(\mathrm{II})-\mathrm{MEPE} / \mathrm{ITO}$ sample remains colorless for several hours (memory effect). However, under ambient conditions, the color gradually turns back to blue; this implies that the sample must be reduced to $\mathrm{Fe}(\mathrm{II})$. The thickness of the spin-coated film is $30-100 \mathrm{~nm}$, as estimated by $\mathrm{CV}$, absorption spectra, and AFM.

The changes in the absorbance of $\mathrm{Fe}$ (II)-MEPE/ITO samples are monitored during the electrochemical cycling in order to study the electrochromic response. Fe-MEPE/ ITO initially exhibits an absorbance at $580 \mathrm{~nm}$, which is assigned to the MLCT transition. The absorption decreases upon oxidation in accordance with a metal centered redox process. Fe(II)-MEPE becomes completely colorless at $1.0 \mathrm{~V}$. The time response is monitored by stepping the potential from 0 to $1.0 \mathrm{~V}$. Evidently, the changes in absorbance observed upon reduction and oxidation are reversible and occur within similar time scales, suggesting that fragmentation or decomposition of the polymer does not occur. Similarly, when Co(II)-MEPE/ITO is oxidized, the absorption at $520 \mathrm{~nm}$ (red), associated with $\mathrm{d}-\mathrm{d}$ transition, decreases upon oxidation. The change in absorbance observed upon reduction and oxidation are reversible between $-0.4 \mathrm{~V}$ and $+0.4 \mathrm{~V}$.

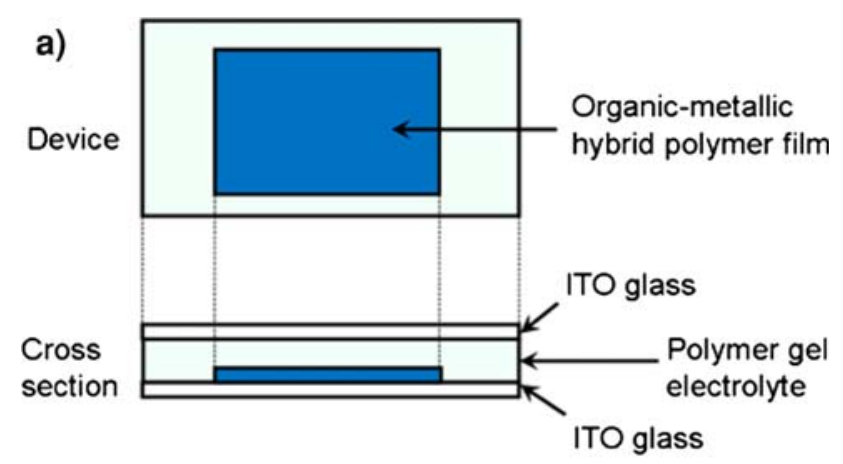

b)

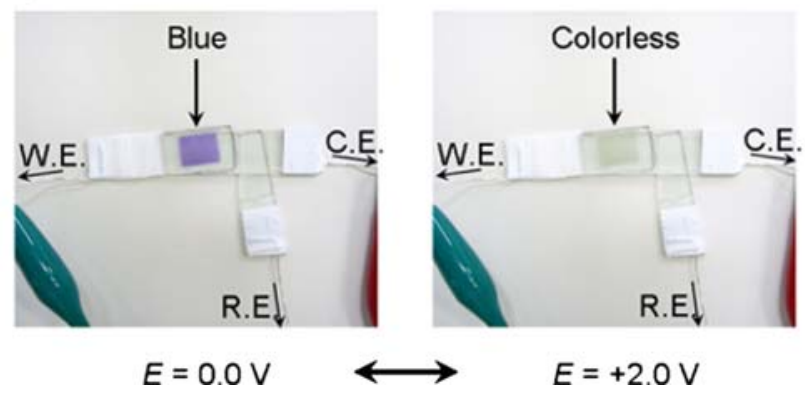

Fig. 2 a Components of an electrochromic solid-state device using a MEPEs film (electrode: ITO; polymer gel electrolyte: polymethylmethacrylate/propylene carbonate/ $\left.\mathrm{LiClO}_{4}\right)$. b Electrochromic behavior of the solid-state device at +3.0 and $-3.0 \mathrm{~V}$ versus $\mathrm{Ag} / \mathrm{Ag}^{+}$
3.4 Solid-State Electrochromic Devices Using Fe(II)and $\mathrm{Fe}(\mathrm{II})-\mathrm{Ru}(\mathrm{II})$-MEPEs

Thus far, we have demonstrated the electrochromic properties of Fe(II)-MEPE in solution; however, solid-state devices that use electrochromic films are advantageous to actual applications such as electronic devices and displays. We have developed a solid-state electrochromic device by using a mixture of polymethylmethacrylate, propylene carbonate, and $\mathrm{LiClO}_{4}$ as a polymer gel electrolyte (Fig. 2a). A film of Fe(II)-MEPE is cast on ITO glass. Color change of the film from colorless to blue is faster (within one-second) than the opposite change (a few seconds), probably because $\mathrm{Fe}(\mathrm{II})$ is more stable than $\mathrm{Fe}(\mathrm{III})$ in the polymer (Fig. 2b).

Furthermore, we fabricate electrochromic solid-state devices with two polymer films as shown in Fig. 3a, which can exhibit different two displays by reversing the direction

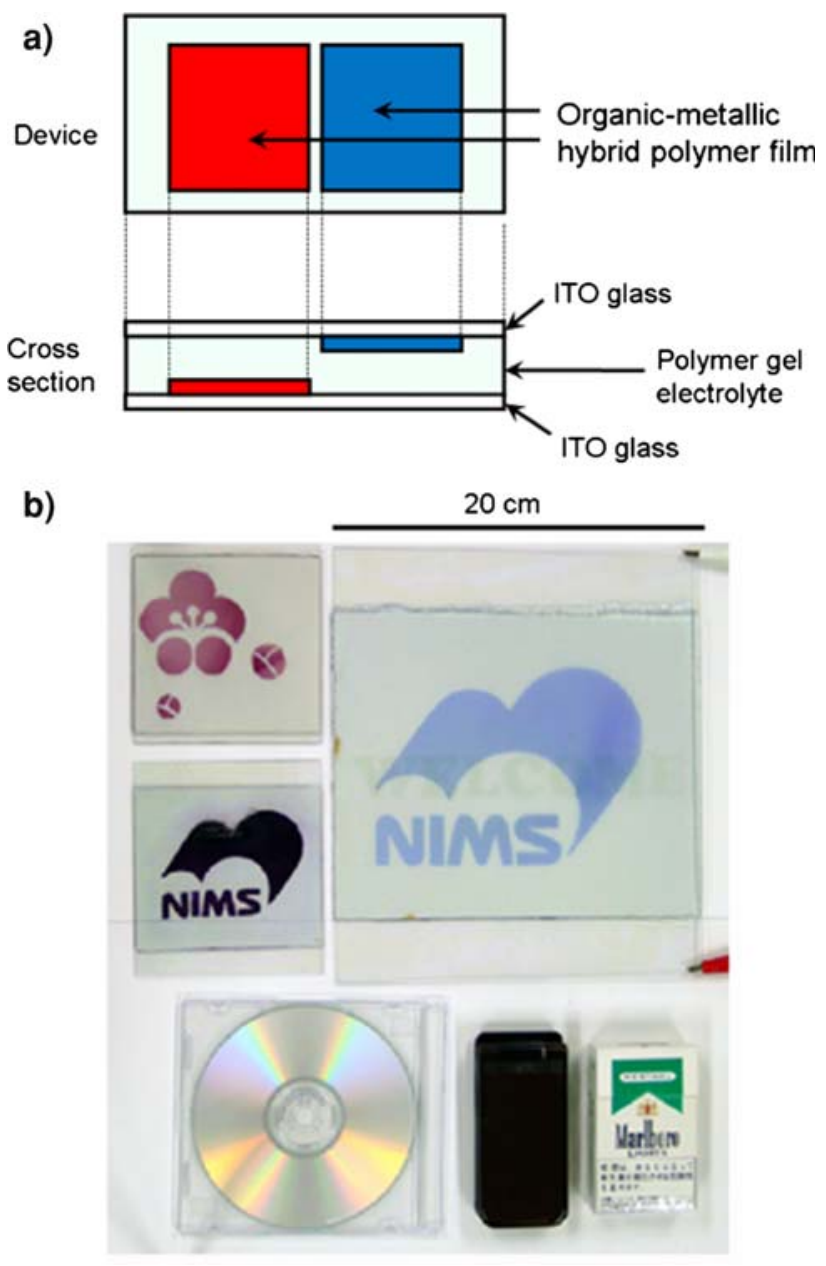

Fig. 3 a Components of an electrochromic solid-state device using two MEPEs films (electrode: ITO; polymer gel electrolyte: polymethylmethacrylate/propylene carbonate/ $\mathrm{LiClO}_{4}$ ). b 5 and 10 inch size of the electrochromic solid-state devices using $\mathrm{Fe}(\mathrm{II})-\mathrm{Ru}(\mathrm{II})$ MEPE and Fe(II)-MEPE 


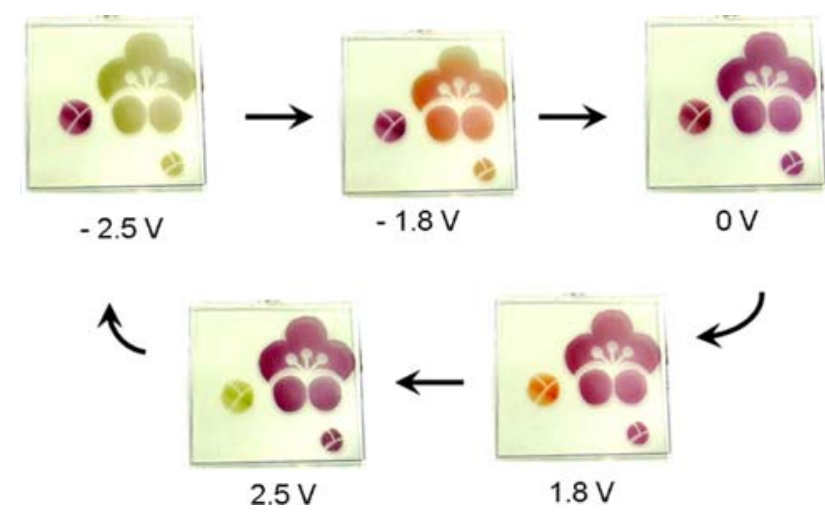

Fig. 4 Multi-color electrochromic properties in the solid-state devices using $\mathrm{Fe}(\mathrm{II})-\mathrm{Ru}(\mathrm{II})-\mathrm{MEPE}$

of current (Fig. 3b). Therefore, the device with two polymer films of $\mathrm{Fe}(\mathrm{II})-\mathrm{Ru}(\mathrm{II})-\mathrm{MEPE}$ exhibits five kinds of displays by changing the applied potentials between $-3 \mathrm{~V}$ and $3 \mathrm{~V}$ (Fig. 4). The redox potentials of $\mathrm{Fe}(\mathrm{II})-\mathrm{Ru}(\mathrm{II})-$ MEPE are 0.77 and $0.92 \mathrm{~V}$ versus $\mathrm{Ag} / \mathrm{Ag}^{+}$as described in Sect. 3.2, but the solid-state device bearing a film of $\mathrm{Fe}(\mathrm{II})-\mathrm{Ru}(\mathrm{II})-\mathrm{MEPE}$ exhibits different colors of red, orange, and pale green, at $0,1.8$, and $2.5 \mathrm{~V}$, respectively. Higher applied potentials than the redox potentials of the polymer are necessary to change the color in the device, because device resistance exists.

\section{Conclusions}

Unlike conventional organic polymers, electrochemical interaction between metal ions and organic moieties in organic-metallic hybrid polymers can produce novel properties. We demonstrated that metallo-supramolecular polyelectrolytes, MEPE, possess unique electrochemical properties. The MLCT is one of the typical interactions between metal ions and the organic ligands. We found that a specific color corresponding to the MLCT absorption is controlled by the electrochemical oxidation/reduction of the metal ions and that this hybrid polymer possesses excellent electrochromic properties. Furthermore, we succeeded to fabricate electrochromic solid-state devices with the hybrid polymers. These materials will be applied to electronic devices and displays such as electronic papers.

Acknowledgement We thank the Ministry of Education, Culture, Sports, Sciences, and Technology, Japan for financial support.

\section{References}

1. J. Roncali, Chem. Rev. 92, 711 (1992)

2. N.C. Greenham, S.C. Moratti, D.D.C. Bradley, R.H. Friend, A.B. Holmes, Nature 365, 628 (1993)

3. D.T. McQuade, A.E. Pullen, T.M. Swager, Chem. Rev. 100, 2537 (2000)

4. C.J. Brabec, N.S. Sariciftci, J.C. Hummelen, Adv. Funct. Mater. 11, 15 (2001)

5. W.U. Huynh, J.J. Dittmer, A.P. Alivisatos, Science 295, 2425 (2002)

6. H. Shirakawa, S. Ikeda, Polym. J. 2, 231 (1971)

7. T. Ito, H. Shirakawa, S. Ikeda, J. Polym. Sci. Pol. Chem. 12, 11 (1974)

8. C.K. Chiang, C.R. Fincher, Y.W. Park, A.J. Heeger, H. Shirakawa, E.J. Louis, S.C. Gau, A.G. Macdiarmid, Phys. Rev. Lett. 39, 1098 (1977)

9. H. Shirakawa, E. J. Louis, A. G. Macdiarmid, C. K. Chiang, A. J. Heeger, J. Chem. Soc. Chem. Commun. 578 (1977)

10. W.P. Su, J.R. Schrieffer, A.J. Heeger, Phys. Rev. Lett. 42, 1698 (1979)

11. T. Yamamoto, A. Morita, Y. Miyazaki, T. Maruyama, H. Wakayama, Z. Zhou, Y. Nakamura, T. Kanbara, S. Sasaki, K. Kubota, Macromolecules 25, 1214 (1992)

12. T. Yamamoto, T. Maruyama, Z.H. Zhou, T. Ito, T. Fukuda, Y. Yoneda, F. Begum, T. Ikeda, S. Sasaki, H. Takezoe, A. Fukuda, K. Kubota, J. Am. Chem. Soc. 116, 4832 (1994)

13. T. Yamamoto, K. Sugiyama, T. Kushida, T. Inoue, T. Kanbara, J. Am. Chem. Soc. 118, 3930 (1996)

14. T. Yamamoto, Z.H. Zhou, T. Kanbara, M. Shimura, K. Kizu, T. Maruyama, Y. Nakamura, T. Fukuda, B.L. Lee, N. Ooba, S. Tomaru, T. Kurihara, T. Kaino, K. Kubota, S. Sasaki, J. Am. Chem. Soc. 118, 10389 (1996)

15. T. Yamamoto, D. Komarudin, M. Arai, B.L. Lee, H. Suganuma, N. Asakawa, Y. Inoue, K. Kubota, S. Sasaki, T. Fukuda, H. Matsuda, J. Am. Chem. Soc. 120, 2047 (1998)

16. H. Hofmeier, U. S. Schubert, Chem. Soc. Rev. 33, 373 (2004) (and references therein)

17. P.R. Andres, U.H. Schubert, Adv. Mater. 16, 1043 (2004)

18. T. Yasuda, I. Yamaguchi, T. Yamamoto, Adv. Mater. 15, 293 (2004)

19. D.G. Kurth, M. Higuchi, Soft Matter 2, 915 (2006) (and references therein)

20. U. Kolb, K. Buscher, C.A. Helm, A. Lindner, A.F. Thunemann, M. Menzel, M. Higuchi, D.G. Kurth, Proc. Natl. Acad. Sci. USA 103, 10202 (2006)

21. M. Higuchi, I. Ikeda, T. Hirao, J. Org. Chem. 62, 1072 (1997)

22. K. Yamamoto, M. Higuchi, S. Shiki, M. Tsuruta, H. Chiba, Nature 415, 509 (2002)

23. M. Higuchi, M. Tsuruta, H. Chiba, S. Shiki, K. Yamamoto, J. Am. Chem. Soc. 125, 9988 (2003)

24. N. Satoh, J.S. Cho, M. Higuchi, K. Yamamoto, J. Am. Chem. Soc. 125, 8104 (2003)

25. M. Higuchi, D.G. Kurth, Chem. Rec. 7, 203 (2007)

26. F. Han, M. Higuchi, D.G. Kurth, Adv. Mater. 19, 3928 (2007)

27. F. Han, M. Higuchi, D.G. Kurth, J. Am. Chem. Soc. 130, 2073 (2008)

28. M. Higuchi, Kobunshi Ronbunshu 65, 399 (2008)

29. F. Han, M. Higuchi, T. Ikeda, Y. Negishi, T. Tsukuda, D.G. Kurth, J. Mater. Chem. 18, 4555 (2008) 